\title{
Autoimmune Hair Loss (Alopecia Areata) Transferred by T Lymphocytes to Human Scalp Explants on SCID Mice
}

\author{
Amos Gilhar, Yehuda Ullmann, Tamara Berkutzki, Bedia Assy, and Richard S. Kalish* \\ Skin Research Laboratory, The Bruce Rappaport Faculty of Medicine, Technion-Israel Institute of Technology, Haifa 31096, Israel; and \\ *Department of Dermatology, State University of New York at Stony Brook, Stony Brook, NY 11794 and Veterans Affairs Medical \\ Center, Northport, New York 11768
}

\begin{abstract}
Alopecia areata is a tissue-restricted autoimmune disease of the hair follicle, which results in hair loss and baldness. It is often psychologically devastating. The role of $\mathrm{T}$ lymphocytes in this disorder was investigated with cell transfer experiments. Scalp explants from patients were transplanted to severe combined immunodeficiency (SCID) mice and injected with autologous $\mathrm{T}$ lymphocytes isolated from involved scalp. $\mathrm{T}$ lymphocytes which had been cultured with hair follicle homogenate along with antigen-presenting cells were capable of inducing the changes of alopecia areata, including hair loss and perifollicular infiltrates of $T$ cells, along with HLA-DR and ICAM-1 expression of the follicular epithelium. Similar changes were not noted in grafts injected with scalp-derived $\mathrm{T}$ cells that had not been cultured with follicular homogenate. These data indicate that alopecia areata is mediated by $\mathrm{T}$ cells which recognize a follicular autoantigen. (J. Clin. Invest. 1998. 101:62-67.) Key words: autoimmunity - hair diseases - intracellular adhesion molecule-1 • CD54 • HLA-DR antigens
\end{abstract}

\section{Introduction}

Alopecia areata is a tissue-restricted autoimmune disease directed at the hair follicle, resulting in hair loss. Patients frequently suffer severe psychiatric consequences (1). This is especially true of girls and young women who become bald. Hair loss is associated with a primarily $\mathrm{CD} 4+$ perifollicular lymphocytic infiltrate (2), along with expression of both DR and ICAM-1 on the follicular epithelium $(3,4)$. Lesional skin from alopecia areata patients grafted onto nude mice regrows hair coincident with a loss of infiltrating lymphocytes from the graft (5). The condition responds to immunosuppressive doses of systemic steroids. Alopecia areata has HLA-DR associations (6), and may coexist with additional autoimmune processes such as autoimmune thyroiditis (7). Autoreactive T cells are present in the scalp infiltrate, but are not specific for alopecia

Address correspondence to Amos Gilhar, M.D., Skin Research Laboratory, Faculty of Medicine, Technion-Israel Institute of Technology, P.O. Box 9649, Haifa 31096, Israel. Phone: 972-4-9841904; FAX: 972-4-8514285; E-mail: gilhar@techunix.technion.ac.il

Received for publication 2 May 1997 and accepted in revised form 29 October 1997.

The Journal of Clinical Investigation

Volume 101, Number 1, January 1998, 62-67

http://www.jci.org areata (8). Circulating autoantibodies to follicular structures are present, but they are also reported in normal controls, and their role in disease pathogenesis is uncertain (9).

This study was undertaken to clarify whether alopecia areata is mediated by $\mathrm{T}$ lymphocytes. Human scalp explants from involved (bald) skin were grafted onto severe combined immunodeficiency (SCID) ${ }^{1}$ mice. Lesional (bald) scalp was used for this study, because transplantation of nonlesional, or normal scalp results in initial hair loss, whereas transplantation of bald skin results in hair regrowth. SCID mice have been used previously to transfer psoriasis to human skin explants with T cell injections $(10,11) .40 \mathrm{~d}$ after transplantation, the scalp explants grew hair, and autologous lymphocytes were injected into the explants in an attempt to reproduce the disease. $\mathrm{T}$ lymphocytes isolated from scalp lesions, and expanded in vitro with homogenates of hair follicles, were able to reproduce the changes of alopecia areata when transferred into scalp explants. T lymphocytes not cultured with hair follicle homogenates did not have this effect. These data strongly indicate a role for $\mathrm{T}$ lymphocytes responding to a follicular autoantigen in the pathogenesis of alopecia areata.

\section{Methods}

Patients. Six patients (five females and one male, mean age $32 \mathrm{yr}$ ) with severe lesions of alopecia areata of 1-15 yr in duration were included in this study. Informed consent was obtained after the nature and possible consequences of the studies were explained. Protocols were approved by the Institutional Review Board. None of the patients had any therapy in the $60 \mathrm{~d}$ before obtaining the scalp biopsy specimens.

19 2-mm punch biopsies from involved areas were obtained from each patient. Four biopsies from each patient were used for the isolation of autologous cutaneous $\mathrm{T}$ cells. Two biopsies were snap-frozen in liquid nitrogen for immunoperoxidase staining. One frozen biopsy was sectioned vertically, and the other was sectioned horizontally. An additional biopsy from each patient was submitted for routine histological examination with hematoxylin and eosin and horizontally sectioned in its entirety. 12 punch biopsies (2-mm) were grafted in sets of three to four SCID mice. This allowed the formation of four treatment groups consisting of six mice, one from each patient.

Animals. SCID mice (Charles River Laboratories, Margate, UK), 2-3 mo old, were used in this study. The mice were raised in the pathogen-free animal facility of the B. Rappaport Faculty of Medicine, Technion-Israel Institute of Technology. Animal care and research protocols were in accordance with institutional guidelines.

Skin grafting. Graft transplantation to SCID mice was performed as described previously (4). Each 2-mm graft was inserted through an

1. Abbreviations used in this paper: scalp TC-Hom, scalp T cells cultured with hair follicle homogenate; SCID, severe combined immunodeficiency. 
incision in the skin into the subcutaneous tissue over the lateral thoracic cage of each mouse, and covered with a standard dressing. The dressing was removed on day 7.

Isolation of T lymphocytes from scalp punch biopsies. Four punch biopsies from each patient were used for $\mathrm{T}$ cell isolation. Tissue infiltrating lymphocytes were isolated from scalp punch biopsy with the use of collagenase, as described previously (12). The number of cells retrieved from each patient averaged 500,000.

Isolation of human PBMC. PBMC were isolated from heparinized whole blood by centrifugation over Hypaque 1077 (Pharmacia Biotech AB, Uppsala, Sweden), and allowed to adhere to 24-well plates (Greiner) in RPMI $1 \%$ human $\mathrm{AB}$ serum for 90 min at $37^{\circ} \mathrm{C}$, $5 \% \mathrm{CO}_{2}$. Nonadherent cells $\left(6-8 \times 10^{6}\right)$ were removed and used for culture.

Hair follicle homogenate preparation. Anagen hair follicles were isolated under a stereodissecting microscope from normal scalp biopsies obtained from healthy subjects who underwent hair transplantation procedures. The hair follicles were mechanically homogenized in PBS using a 15-ml glass tissue grinder (Lawson Mardon Wheaton, Milville, NJ), centrifuged at $500 \mathrm{~g}$ for $30 \mathrm{~min}$, and sterilized by millipore filtration ( $0.22 \mu \mathrm{m}$; Gelman Sciences, Inc., Ann Arbor, MI). The protein content of each homogenate was assayed by a colorimetric assay (13). The samples were stored at $-20^{\circ} \mathrm{C}$ and diluted with culture medium to a final protein concentration of $10 \mu \mathrm{g} / \mathrm{ml}$.

Culture of T lymphocytes. Lymphocytes were plated at $10^{5}$ cells/ well in RPMI-FCS, along with irradiated (5000R) PBMC $\left(10^{5}\right.$ cells/ well) in 24-plate wells (Greiner Labortecnik, Frichenhausen, Germany), as feeders. After $3 \mathrm{~d}$ of culture, rIL-2 (10 U/ml; Genzyme Corp., Cambridge, MA) was added. Every $5 \mathrm{~d}$, the $\mathrm{T}$ cells were restimulated with feeders, for a total of five cycles over $5 \mathrm{wk}$.

Lymphocytes were stimulated with follicular homogenate preparations by a modification of the above protocol, in which follicular homogenate $(10 \mu \mathrm{g} / \mathrm{ml})$ was added along with the feeder cells at each stimulation.

Phenotypic characterization of cultured T lymphocytes. Phenotype of the cultured of $\mathrm{T}$ cell lines was determined by cytofluorograph analysis (FACScalibur ${ }^{\circledR}$; Becton Dickinson, Mountain View, CA). The following mAbs were used: anti-CD3 FITC (clone SK 7; Becton Dickinson), anti-CD4 (IgG2a, S3.5), anti-CD8 (IgG2a, 3B5) (Caltag Labs, South San Francisco, CA), anti-HLA-DR (IgG2a, DK22; DAKO A/S, Glostrup, Denmark). Subtype-matched mouse IgGFITC (Becton Dickinson) was used as a negative control.

Injection of cultured T lymphocytes into grafts on SCID mice. The SCID mice bearing grafts of lesional scalp were divided into four groups of six mice each. On day 40 the grafts were injected intradermally with autologous lymphocytes as noted below. The first group received intradermal injections of autologous PBMC into the scalp grafts. Each graft received $1.6 \times 10^{6}$ cells in $0.2 \mathrm{ml}$ RPMI 1640 . The grafts of the second group were injected intradermally with $1.3 \times 10^{6}$ autologous T cells (in $0.2 \mathrm{ml}$ RPMI 1640) isolated from lesional scalp (scalp T cells). Before injections the T cells were cultured with hair follicle homogenates as an antigen. The third group was also injected intradermally with autologous $\mathrm{T}$ cells of lesional scalp but not cultured with homogenates. These grafts received the same number of cells as were injected into the second group $\left(1.3 \times 10^{6}\right.$ cells $)$. The fourth group served as a control, and was not injected with $\mathrm{T}$ cells, in order to observe a possible effect of the residual human $\mathrm{T}$ cells within the grafts. On day 82, the mice were killed and grafts were harvested for immunohistochemical and histological analysis.

Immunohistochemical staining. Staining was performed as reported previously (14). mAbs to human antigens used were as follows: anti-HLA-DR, anti-CD54 (ICAM-1) (Biodesign International, Kennebunk, ME), anti-CD3, anti-CD4, anti-CD8, anti-CD25 (DAKO $\mathrm{A} / \mathrm{S}$ ), and anti-HLA-class 1 (DAKO A/S). Each specimen was coded at the time of biopsy and evaluated by two observers who were blind to the coding.

Statistical analysis. Statistical analysis was carried out using ANOVA and the Tukey-Kramer multiple comparisons test.
Table I. Recovery of Scalp T Cells from Cultures with and without Hair Follicular Homogenate

\begin{tabular}{llll}
\hline \multicolumn{1}{c}{ Conditions } & Initial No. & \multicolumn{1}{c}{ Final No. } & HLA-DR \\
\hline IL-2 & $0.60 \times 10^{6}(0.1)$ & $1.5 \times 10^{6}(0.7)$ & $19 \%$ \\
IL-2 + Hom* & $0.56 \times 10^{6}(0.1)$ & $9.6 \times 10^{6}(0.7)^{\ddagger}$ & $84.5 \%$
\end{tabular}

* Scalp T cells cultured with hair follicle homogenate in the presence of antigen-presenting cells. ${ }^{\ddagger} P<0.001$ by ANOVA.

\section{Results}

T cells were cultured from scalp biopsies of alopecia areata patients with the use of irradiated autologous PBMC and hair follicle homogenate, as described above. Flow cytometric analysis of the scalp $\mathrm{T}$ cells before injections revealed the following phenotype: CD3+ 98\%, CD4+ 55.7\%, CD8+ 84.5\%, and HLA-DR $+84.5 \%$. This reflects the presence of double-positive CD4+CD8+ cells. Double-positive CD4+CD8+ cells are commonly found after culture of skin infiltrating cells (12). A similar T cell phenotype was found for scalp TC-homogenate (scalp TC-Hom) and scalp T cells, with the exception that the percentage of HLA-DR+ was $19 \%$ for T cells cultured without homogenate (Table I). There was also a significantly higher recovery of $\mathrm{T}$ cells from cultures with hair follicle homogenate relative to scalp $\mathrm{T}$ cell cultures without homogenate (Table I, $P<0.001$ by ANOVA). The increases in T cell recovery and HLA-DR expression indicate activation and proliferation of the scalp $\mathrm{T}$ cells by follicular homogenate. The analysis of cultured PBMC before injections revealed the following phenotype: CD3+ 99\%, CD4+ 15.6\%, CD8+ 64.8\%, and HLA-DR $+48.8 \%$. All T cells had been cultured under identical conditions before injection, with the exception that scalp TC-Hom also received follicular homogenate.

The biopsies obtained from lesional scalp before grafting were characteristic of alopecia areata. No terminal hairs were observed, and some follicles were observed to be in the anagen phase. Perifollicular mononuclear cell infiltrates were associated with $48 \%$ of hair follicles. The perifollicular infiltrates were composed of both CD4+ and CD8 $+\mathrm{T}$ cells, with a relative predominance of $\mathrm{CD} 4+$ over CD8+ cells. CD4+ cells were found around the hair follicles. In contrast, intrafollicular infiltrates were composed mostly of CD8+ cells, with only a few

Table II. Injection of Scalp T Cells Incubated with Follicular Homogenate Induced Expression of ICAM-1 and HLA-DR

\begin{tabular}{|c|c|c|}
\hline Treatment & HLA-DR & ICAM-1 \\
\hline Before transplant & $18 \%(19 / 105)^{*}$ & $36 \%(39 / 94)$ \\
\hline Noninjected & $0 \%(0 / 58)$ & $0 \%(0 / 49)$ \\
\hline PBMC injected & $0 \%(0 / 39)$ & $9 \%(3 / 34)$ \\
\hline Scalp T cells & $8 \%(6 / 62)$ & $0 \%(0 / 49)$ \\
\hline Scalp TC-Hom & $31 \%(15 / 45)$ & $48 \%(21 / 43)$ \\
\hline
\end{tabular}

Alopecia areata scalp biopsies were transplanted to SCID mice, and injected intradermally with autologous cultured $\mathrm{T}$ cells $40 \mathrm{~d}$ later. Grafts were harvested at $82 \mathrm{~d}$ and analyzed by immunochemistry. *Number of follicles expressing antigen/total number of follicles (\%). ${ }^{\ddagger}$ Injected with scalp $\mathrm{T}$ cells cultured with hair follicle homogenate in the presence of antigen-presenting cells. 

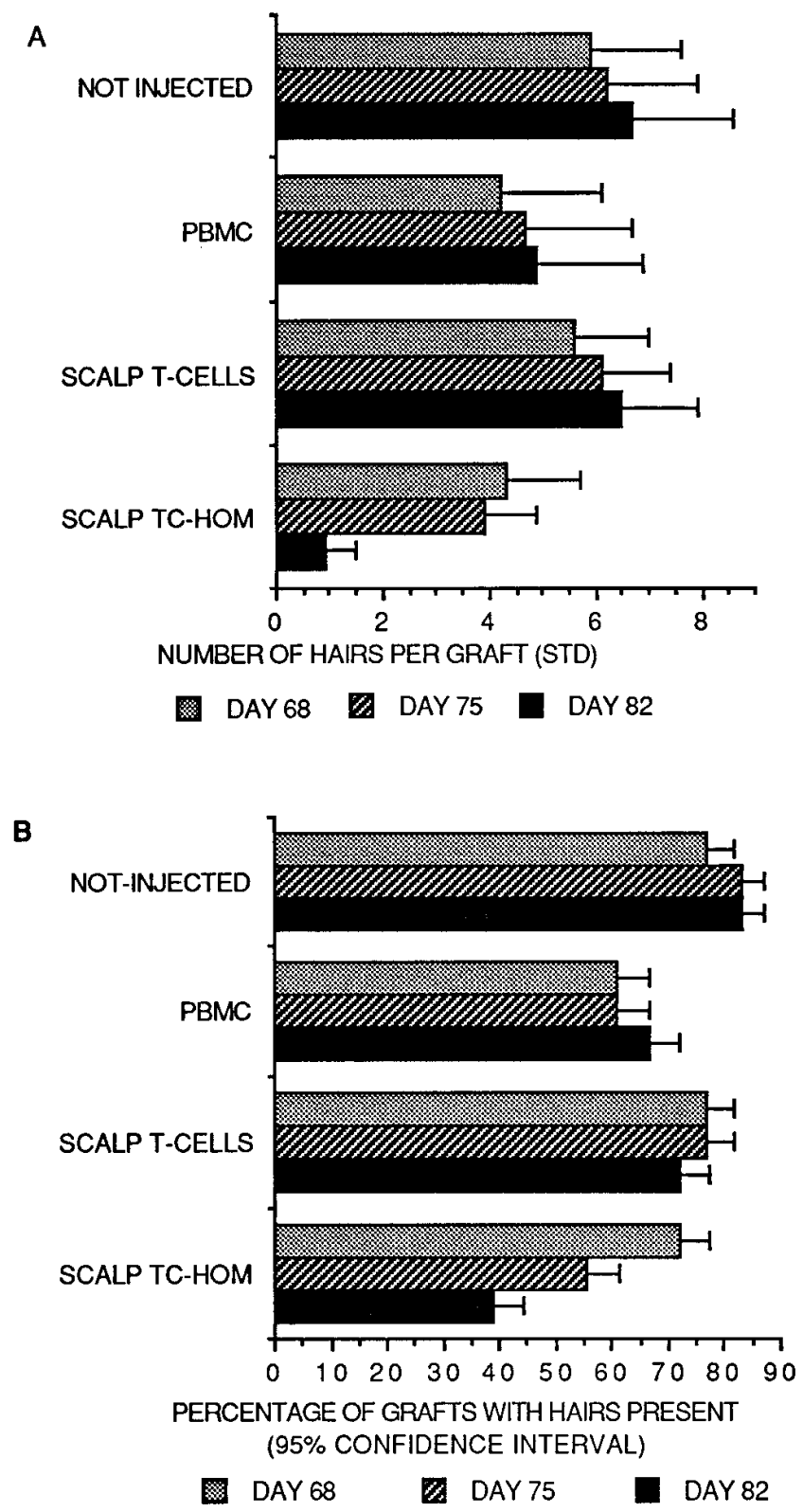

Figure 1. The grafts were injected intradermally with T cells as indicated on day 40. ( $A$ ) Number of hairs per graft ( $n=18$ grafts). On day 82 , the difference between scalp TC-Hom and the other treatments was statistically significant (ANOVA, $P<0.001$ ). (B) Percentage of grafts with hair on days 68,75 , and 82 after engraftment onto SCID mice ( $n=18$ grafts).

CD4+ cells detected. IL-2 receptor (CD25) expression was detected among the cells of both the peri- and intrafollicular infiltrates. HLA-DR and ICAM-1 were expressed by 18 and $36 \%$ of the follicles before grafting (Table II).

The number of hairs in each graft was determined under magnification $(\times 5)$ twice weekly. By day 40, when lymphocytes were injected, hair was present in $38 \%$ of the grafts. Hair growth increased in all treatment groups up to $68 \mathrm{~d}$ (Fig. 1). By day 82 , hair was present in $83 \%$ of noninjected control grafts (Fig. 1). Similar continual increases in hair growth were observed in grafts injected with PBMC, as well as scalp T cells. In contrast, scalp grafts injected with scalp T cells which had been incubated with hair follicle homogenate lost hair after $68 \mathrm{~d}$ (Fig. 1). Thus, scalp TC-Hom induced hair loss. Injection of these $\mathrm{T}$ cells also reduced the number of hairs per graft (Fig. 1). The number of hairs per graft on days 75 and 82 was significantly (ANOVA, $P<0.05$ ) lower in grafts injected with scalp TC-Hom than in the remaining groups. Grafts injected with scalp TC-Hom also had a significant reduction in hairs per graft on day 82 relative to day 68 (ANOVA, $P<0.05$ ).

Grafts treated with scalp TC-Hom had histologic evidence of alopecia areata (Fig. 2), including a dense infiltrate of mononuclear cells, as well as follicular dystrophy, with matrix and hair bulb degeneration. Few anagen follicles were present only at higher levels of the dermis, and no terminal hairs were noted. In contrast, grafts injected with PBMC, or scalp T cells (no homogenate), revealed normal terminal hairs without follicular damage (Fig. 2).

The infiltrates of grafts injected with scalp TC-Hom were composed of both CD4+ and CD8+ cells. CD4+ cells were primarily perifollicular, whereas CD8+ cells were intrafollicular. In contrast, grafts injected with PBMC or scalp T cells (cultured without homogenate) exhibited rare CD8+ cells and moderate perifollicular infiltrates in only $24-27 \%$ of hair follicles.

Foci of CD25+ cells were detected in the upper dermis as well as around and within 11 out of $42(26 \%)$ hair follicles of grafts treated with scalp TC-Hom. PBMC and scalp T cellinjected grafts had foci of CD25+ cells in the upper dermis only.

HLA-DR and ICAM-1 expression was induced by injection of scalp TC-Hom (Table II and Figs. 3 and 4). Similar induction of HLA-DR and ICAM-1 expression was not observed with injection of scalp $\mathrm{T}$ cells not incubated with hair follicle homogenate.

\section{Discussion}

These data strongly support a role for T cells in the pathogenesis of alopecia areata. In these experiments, we first caused a remission of alopecia areata, and then induced a recurrence. Since alopecia areata is naturally a remitting, recurring condition this model mimics the natural pathology of the disease. Alopecia areata was reproduced in grafts injected with autologous $\mathrm{T}$ cells that had been isolated from patient scalps and cultured with hair follicle homogenates. Characteristics of alopecia areata which were reproduced included hair loss, a dense perifollicular T cell infiltrate, and expression of HLA-DR, and ICAM-1 by follicular epithelium. Grafts injected with PBMC, or scalp T cells that had not been cultured with hair follicle homogenate, did not induce these changes. The requirement for follicular homogenate in the activation of the pathogenic $\mathrm{T}$ cells suggests that $\mathrm{T}$ cell recognition of follicular autoantigen is central to the pathogenesis of alopecia areata.

The follicular homogenate was obtained from normal scalp and washed out before injection of the T cells. It is proposed that the action of the follicular homogenate was to selectively expand autoantigen reactive $T$ cells. The inability of the scalp $\mathrm{T}$ cells to produce pathology in the absence of follicular homogenate may reflect a low frequency of autoreactive $\mathrm{T}$ cells in the absence of antigen-specific stimulation.

It was necessary to use lesional (bald) scalp for the explants because transplantation of noninvolved scalp, or scalp from 

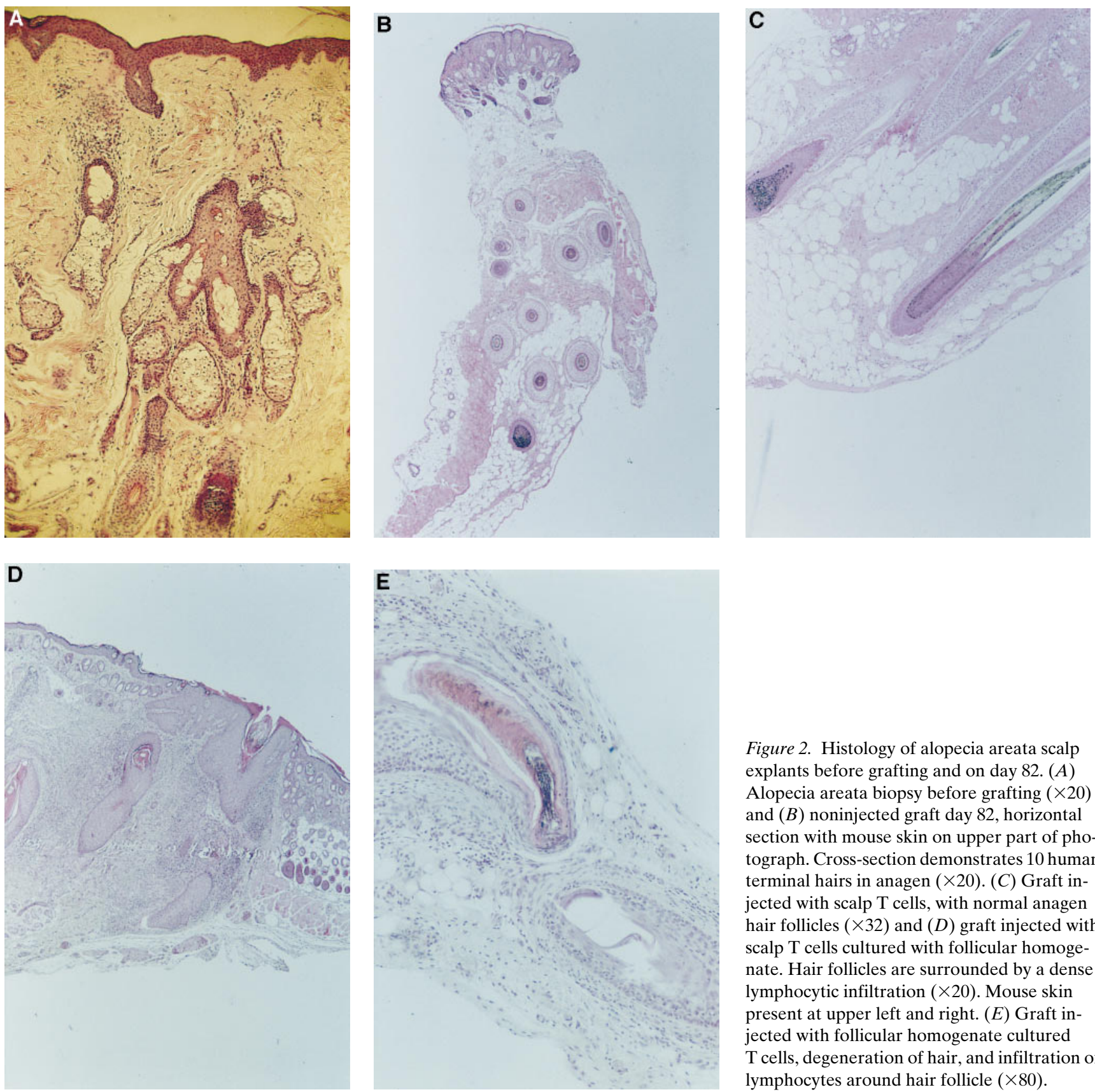

Figure 2. Histology of alopecia areata scalp explants before grafting and on day 82. $(A)$ Alopecia areata biopsy before grafting $(\times 20)$ and $(B)$ noninjected graft day 82 , horizontal section with mouse skin on upper part of photograph. Cross-section demonstrates 10 human terminal hairs in anagen $(\times 20)$. $(C)$ Graft injected with scalp $T$ cells, with normal anagen hair follicles $(\times 32)$ and $(D)$ graft injected with scalp $\mathrm{T}$ cells cultured with follicular homogenate. Hair follicles are surrounded by a dense lymphocytic infiltration $(\times 20)$. Mouse skin present at upper left and right. (E) Graft injected with follicular homogenate cultured T cells, degeneration of hair, and infiltration of lymphocytes around hair follicle $(\times 80)$.

normal donors results in loss of hair without regrowth (15). For this reason, it was not possible to perform these experiments with noninvolved or normal scalp explants. Previously, we demonstrated normal hair growth in scalp biopsies of patients with alopecia areata transplanted onto nude mice (5), indicating that hair follicles of alopecia areata have growth potential. The experiments were designed so that $\mathrm{T}$ lymphocytes were always injected into autologous scalp explants, thereby ensuring MHC compatibility.

The ability of injected $\mathrm{T}$ cells to induce alopecia areata was not a nonspecific effect of activated $\mathrm{T}$ cells, since it was not produced by the injection of IL-2-activated $\mathrm{T}$ cells isolated from peripheral blood or scalp. Prior experiments have demonstrated that lesional scalp T cells activated with PHA were not capable of inducing alopecia areata, indicating this is not a nonspecific effect of activated $\mathrm{T}$ lymphocytes (data not shown). The role for $\mathrm{T}$ cells in alopecia areata is supported by the failure of administration of patients' serum to inhibit hair growth in the nude mouse model (16).

In common with a number of tissue-restricted autoimmune disorders, lesional hair follicles in alopecia areata show ectopic HLA-DR and ICAM-1 molecules (17). These markers are known to be important in antigen presentation and lymphocyte trafficking and can be modulated by the action of cytokines including IFN- $\gamma$.

Elevated HLA class I expression in follicular epithelium is associated with CD8+ intrafollicular infiltrates (18). This may reflect CD8+ cell recognition of hair follicle autoantigens pre- 


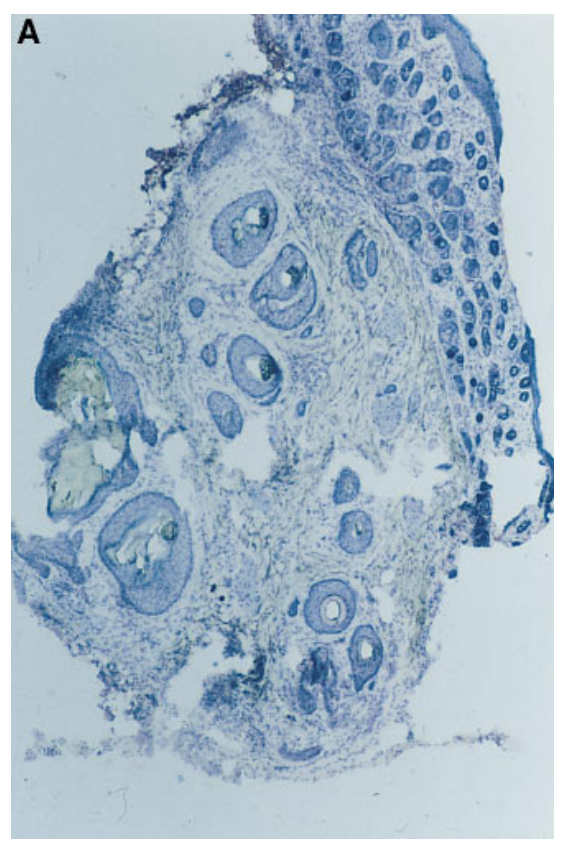

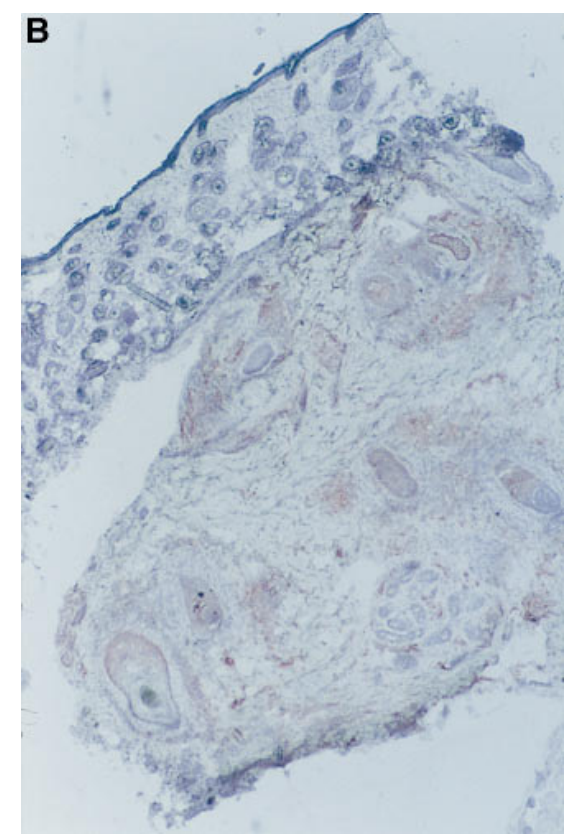

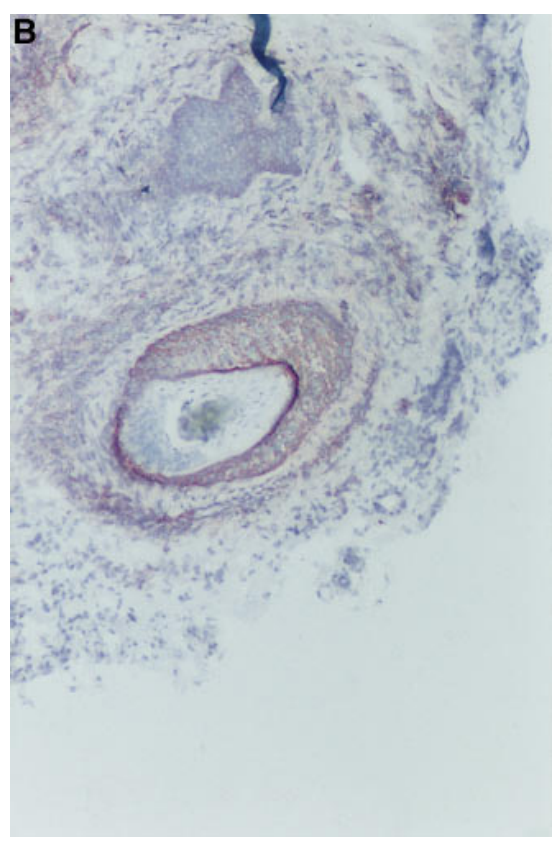

Figure 3. Expression of HLA-DR on scalp explants, day 82. (A) Graft injected with scalp T cells $(\times 32)$. $(B)$ Graft injected with scalp $\mathrm{T}$ cells cultured with follicular homogenate $(\times 32)$.

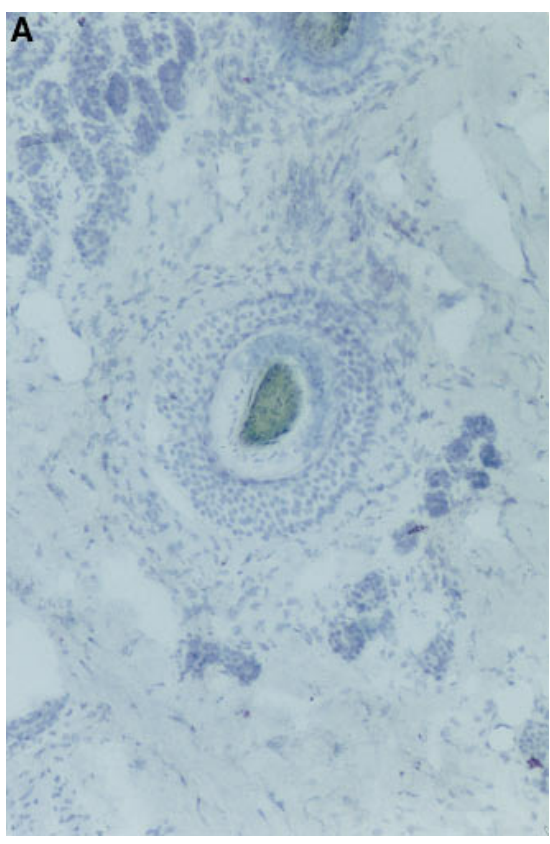

Figure 4. Expression of ICAM-1 on scalp explants, day 82. (A) Graft injected with scalp T cells $(\times 80)$. $(B)$ Graft injected with scalp T cells cultured with follicular homogenate $(\times 80)$. sented by HLA class I $(19,20)$. Dense perifollicular infiltrates of CD4+ cells were also noted in association with dystrophic follicles. Autoreactive CD4+ cells, which recognize autologous monocytes, are found in the infiltrate of alopecia areata and are proposed to have a secondary role in amplification of the pathology (8).

T cells isolated from scalps of patients with alopecia areata and cultured with hair follicle homogenates induced both hair loss and the pathologic findings of alopecia areata when injected into autologous scalp grafted onto SCID mice. These data indicate that alopecia areata is a tissue-restricted autoimmune disease, mediated by a $\mathrm{T}$ lymphocyte response to a follicular autoantigen.

\section{Acknowledgments}

This work was partially supported by a grant from the National Alopecia Areata Foundation.

\section{References}

1. Colon, E.A., M.K. Popkin, A.L. Callies, N.J. Dessert, and M.K. Hordkinsky. 1991. Lifetime prevalence of psychiatric disorders in patients with alopecia areata. Compr. Psychiatry. 32:245-251.

2. Todes-Taylor, N., R. Turner, G.S. Wood, P.T. Stratte, and V.B. Morhenn. 1984. T cell subpopulations in alopecia areata. J. Am. Acad. Dermatol. 11:216-223.

3. Messenger, A.G., and S.S. Bleehen. 1985. Expression of HLA-DR by anagen hair follicles in alopecia areata. J. Invest. Dermatol. 85:569-572. 
4. Khoury, E.L., V.H. Price, and J.S. Greenspan. 1988. HLA-DR expression by hair follicle keratinocytes in alopecia areata: evidence that it is secondary to the lymphoid infiltration. J. Invest. Dermatol. 90:193-200.

5. Gilhar, A., and G.G. Krueger. 1987. Hair growth in scalp grafts from patients with alopecia areata and alopecia universalis grafted onto nude mice. Arch. Dermatol. 123:44-50.

6. Duvic, M., M.K. Hordinsky, V.C. Fiedler, W.R. O'Brien, R. Young, and J.D. Reveille. 1991. HLA-D locus associations in alopecia areata. Arch. Dermatol. 127:64-68.

7. Milgraum, S.S., A.J. Mitchell, G.E. Bacon, and J.E. Rasmussen. 1987. Alopecia areata, endocrine function, and autoantibodies in patients 16 years of age or younger. J. Am. Acad. Dermatol. 17:57-61.

8. Kalish, R.S., K.L. Johnson, and M.K. Hordinsky. 1992. Alopecia areata: autoreactive $\mathrm{T}$ cells are variably enriched in scalp lesions relative to peripheral blood. Arch. Dermatol. 128:1072-1077.

9. Tobin, D.J., S.K. Hann, M.S. Song, and J.C. Bystryn. 1997. Hair follicle structures targeted by antibodies in patients with alopecia areata. Arch. Dermatol. 133:57-61.

10. Boehncke, W.H., D. Dressel, T.M. Zollner, and R. Kaufmann. 1996. Pulling the trigger on psoriasis. Nature. 379:777.

11. Wrone-Smith, T., and B.J. Nickoloff. 1996. Dermal injection of immunocytes induces psoriasis. J. Clin. Invest. 98:1878-1887.

12. Kalish, R.S., and K.L. Johnson. 1990. Enrichment and function of urushiol (poison ivy) specific T-lymphocytes in lesions of allergic contact dermatitis to urushiol. J. Immunol. 145:3706-3713.

13. Bradford, M.M. 1976. A rapid and sensitive method for the quantitation of microgram quantities of protein utilizing the principle of protein-dye binding. Anal. Biochem. 72:148-154.

14. Gilhar, A., T. Pillar, M. David, and S. Eidelman. 1991. Melanocytes and Langerhans cells in aged versus young skin before and after transplantation onto nude mice. J. Invest. Dermatol. 96:210-214.

15. Gilhar, A., T. Pillar, and A. Etzioni. 1988. The effect of topical cyclosporin on the immediate shedding of human scalp hair grafted onto nude mice. Br. J. Dermatol. 119:767-770.

16. Gilhar, A., T. Pillar, B. Assy, and M. David. 1992. Failure of passive transfer of serum from patients with alopecia areata and alopecia universalis to inhibit hair growth in transplants of human scalp skin grafted on to nude mice. Br. J. Dermatol. 126:166-171.

17. Nickoloff, B.J., and C.E.M. Griffiths. 1991. Aberrant intercellular adhesion molecule-1 expression by hair follicle epithelial cells and endothelial leukocyte adhesion molecule- 1 by vascular cells are important adhesion-molecule alterations in alopecia areata. J. Invest. Dermatol. 96:91S-92S.

18. Peret, C., L. Weisner-Menzel, and R. Haple. 1984. Immunohistochemical analysis of T-cell subsets in the peribulbar and intrabulbar infiltrates of alopecia areata. Acta. Derm. Venereol. (Stockh.). 64:26-30.

19. Paus, R., A. Slominski, and B.M. Czarnetzki. 1994. Is alopecia areata an autoimmune-response against melanogenesis-related proteins, exposed by abnormal MHC class I expression in the anagen hair bulb? Yale J. Biol. Med. 66: 541-554.

20. McElwee, K.K.K.J., E.M. Spiers, and R.F. Oliver. 1996. In vivo depletion of CD8 + T cells restores hair growth in the DEBR model for alopecia areata. Br. J. Dermatol. 135:211-217. 